\title{
Trombólise intra-arterial e controle da hipotensão em acidente vascular cerebral
}

\author{
Thrombolysis and control of hypotension in stroke
}

\section{Fernando Morgadinho Santos Coelho' ${ }^{1}$, Tania Oliveira Lopes² Mauricio Bernstein ${ }^{3}$, Antonio Capone Neto ${ }^{4}$, Eduardo Noda Kihara ${ }^{5}$, Marcio Martines dos Santos ${ }^{6}$, Daniela Santos Medeiros da Silva ${ }^{7}$, Cláudio Luiz Lottemberg ${ }^{8}$}

\section{RESUMO}

Paciente de 83 anos, feminina, com acidente vascular cerebral isquêmico por obstrução da artéria cerebral média direita. Realizada trombólise intra-arterial após cinco horas de evolução com controle da hipotensão usando noradrenalina obtendo sucesso na evolução e acompanhamento. Um aumento da morbidade e mortalidade em pacientes com hipotensão tem sido relatado. $\mathrm{O}$ controle da hipotensão arterial em pacientes com AVC é uma das medidas importantes na fase aguda da doença para uma boa evolução.

Unitermos. Acidente Cerebral Vascular, Norepinefrina, Terapia Trombolítica

Citação. Coelho FMS, Lopes TO, Bernstein M, Capone Neto A, Kihara EN, Santos MM, Silva DSM, Lottemberg CL. Trombólise intra-arterial e controle da hipotensão em acidente vascular cerebral.

Trabalho realizado no Programa de Neurologia, Prática Médica, do Hospital Israelita Albert Einstein, São Paulo-SP, Brasil.

1.Neurologista, Mestre, Hospital Israelita Albert Einstein, São Paulo-SP, Brasil.

2.Enfermeira, Professora da Faculdade de Enfermagem do Hospital Israelita Albert Einstein, São Paulo-SP, Brasil.

3.Cardiologista, Doutor pelo Incor, Hospital Israelita Albert Einstein, São Paulo-SP, Brasil.

4.Intensivista, Doutor pela Unicamp, Hospital Israelita Albert Einstein, São Paulo-SP, Brasil.

.Hemodinamicista, Doutor, Setor de Radiologia Vascular do Hospital Israelita Albert Einstein, São Paulo-SP, Brasil.

6.Anestesista, Hospital Israelita Albert Einstein, São Paulo-SP, Brasil. Anestesista, Mestre, Hospital Israelita Albert Einstein, São Paulo-SP, Brasil.

7.Oftalmologista, Doutor pela Unifesp, Hospital Israelita Albert Einstein, São Paulo-SP, Brasil.

\section{SUMMARY}

83 years, women, with ischemic stroke by obstruction of the right middle cerebral artery. Thrombolysis after five hours of onset and control of the hypotension with norepinephrine were done successfully. Increase in morbidity and mortality in patients with hypotension has been reported. The control of hypotension in patients with stroke is important measure in the acute phase of the disease for good development.

Keywords. Stroke, Norepinephrine, Thrombolysis.

Citação. Coelho FMS, Lopes TO, Bernstein M, Capone Neto A, Kihara EN, Santos MM, Silva DSM, Lottemberg CL. Thrombolysis with control of hypotension in stroke.

Endereço para correspondência:

Fernando MS Coelho

R. Xavier Curado, 351/204

CEP 04210-100, São Paulo-SP, Brasil.

Tel. 1137471312

Email: fernandomorgadinho@hotmail.com

Recebido em: 21/01/2008 Revisado em: 22/01/2008 a 05/03/2008

Aceito em: 06/03/2008

Conflito de interesses: não 


\section{INTRODUÇÃO}

O controle da pressão arterial sistêmica (PA) na fase aguda do acidente vascular cerebral isquêmico (AVCl) é consenso na literatura mundial ${ }^{1-4}$. $O$ controle de hipertensão arterial em pacientes na fase aguda do $\mathrm{AVCl}$ com indicação de trombólise sistêmica e intra-arterial tem sido recomendado1. O manejo intensivo da PA de 15 em 15 minutos nas primeiras 2 horas, de 30 em 30 minutos nas primeiras 6 horas e de 60 em 60 minutos nas primeiras 24 horas é recomendação para boa prática médica e melhor evolução do paciente 2 .

A morbimortalidade de pacientes com AVCI aumenta com hipertensão como também com hipotensão arterial. $\mathrm{O}$ risco relativo de morbidade e mortalidade em pacientes tanto com PA acima de $220 \mathrm{mmHg}$ como abaixo de $150 \mathrm{mmHg}$ é de 2,6. Portanto, é tão prejudicial ao paciente com AVCI na fase aguda manter níveis pressóricos elevados quanto baixos ${ }^{3}$.

Recentemente a literatura mundial vem abordando o tema da hipertensão arterial induzida em pacientes com AVCI na fase aguda. O mecanismo de defesa do organismo contra uma injúria isquêmica cerebral é o aumento reflexo da PA para melhora da região mal perfundida. $O$ aumento excessivo da PA pode ser prejudicial principalmente em casos de indicação de trombólise por risco de hemorragias. Porém, há casos em que o mecanismo de aumento da PA reflexa não é eficaz por disfunção da autorregulação cerebral como nesse caso, com lesão de insula direita ${ }^{5}$. Com a manutenção da hipoperfusão por hipotensão arterial, a área perilesionada (penumbra) evolui rapidamente com morte celular e aumento do território isquêmico total ${ }^{6}$.

Algumas medidas para se evitar o efeito deletério da hipotensão relativa na fase aguda do AVCI têm sido descritas. A simples suplementação de volume, seja por infusão de cristalóides ou colóides, não altera a morbidade ou mortalidade. $\mathrm{O}$ uso de epinefrina demonstrou pior evolução por maiores complicações cardiovasculares. Poucos relatos têm sido vistos com o uso de noradrenalina e dobutamina. A droga de escolha é a fenilefrina com ação seletiva alfa 1 em artérias cerebrais e pouca ação em vasos coronarianos, embora com risco maior em induzir bradicardias $s^{6,7}$.

O objetivo desse artigo é descrever um caso de AVCI tratado na fase aguda com noradrenalina após trombólise intra-arterial, com boa evolução.
Obteve-se autorização para esta publicação por familiares e pela própria paciente.

\section{RELATO DE CASO}

Paciente de 83 anos, feminina, com antecedentes de hipertensão arterial sistêmica e osteoartrose. Foi encontrada caída em casa às $19 \mathrm{~h}$ com um último contato pelo telefone ao redor das $17 \mathrm{~h}$ sem anormalidades. Havia relato dos parentes de uma dificuldade de articulação da fala, de um desvio do olhar e da cabeça para direita e de uma dificuldade de movimentação do dimídio esquerdo.

Foi admitida no Primeiro Atendimento ao redor das $20 \mathrm{~h}$ com hemiparesia completa proporcionada grau 2 à esquerda, com desvio do olhar conjugado para direita, disártrica, com hemihipoestesia superficial em dimídio esquerdo, hemianopsia homônima à esquerda, pupilas isocóricas e fotorreagentes, sem rigidez de nuca, com escala de $\mathrm{NIH}$ de 188. Os sinais vitais: PA $170 \times 80 \mathrm{mmHg}$, freqüência cardíaca de 100 bpm e glicemia capilar igual a 150 $\mathrm{mg} / \mathrm{dl}$.

O eletrocardiograma evidenciava fibrilação atrial. A tomografia computadorizada de crânio realizada sem contraste com 4 horas do ictus foi interpretada como normal (Figura 1). Imediatamente após, a paciente foi encaminhada para ressonância nuclear magnética com 4 horas e meia de evolução (Figura 2). Durante sedação para Ressonância evoluiu com hipotensão arterial, mantendo a fibrilação atrial com resposta ventricular ao redor de $115 \mathrm{bpm}$ e PA de 100/75 mmHg quando foi ventilada e hidratada. Optou-se por introduzir com noradrenalina endovenosa com o objetivo de manter a PA média acima de $90 \mathrm{mmHg}$. A Ressonância Magnética por difusão mostrou imagem desproporcional em relação ao quadro clínico observado. Realizada angiografia cerebral com monitorização intensiva da PA durante o procedimento hemodinâmico. Realizado cateterismo que evidenciou obstrução do ramo M1 da artéria cerebral média à direita com abertura total do vaso após $10 \mathrm{mg}$ de alteplase intra-arterial (Figura 3).

Após 45 minutos da angiografia cerebral a paciente foi submetida à tomografia de crânio sem contraste (Figura 4). Foi encaminhada para terapia intensiva com sedação contínua, noradrenalina mantendo PA média entre 80 e $95 \mathrm{mmHg}$, além de heparinização precoce e programação para reversão da fibrilação arterial. 

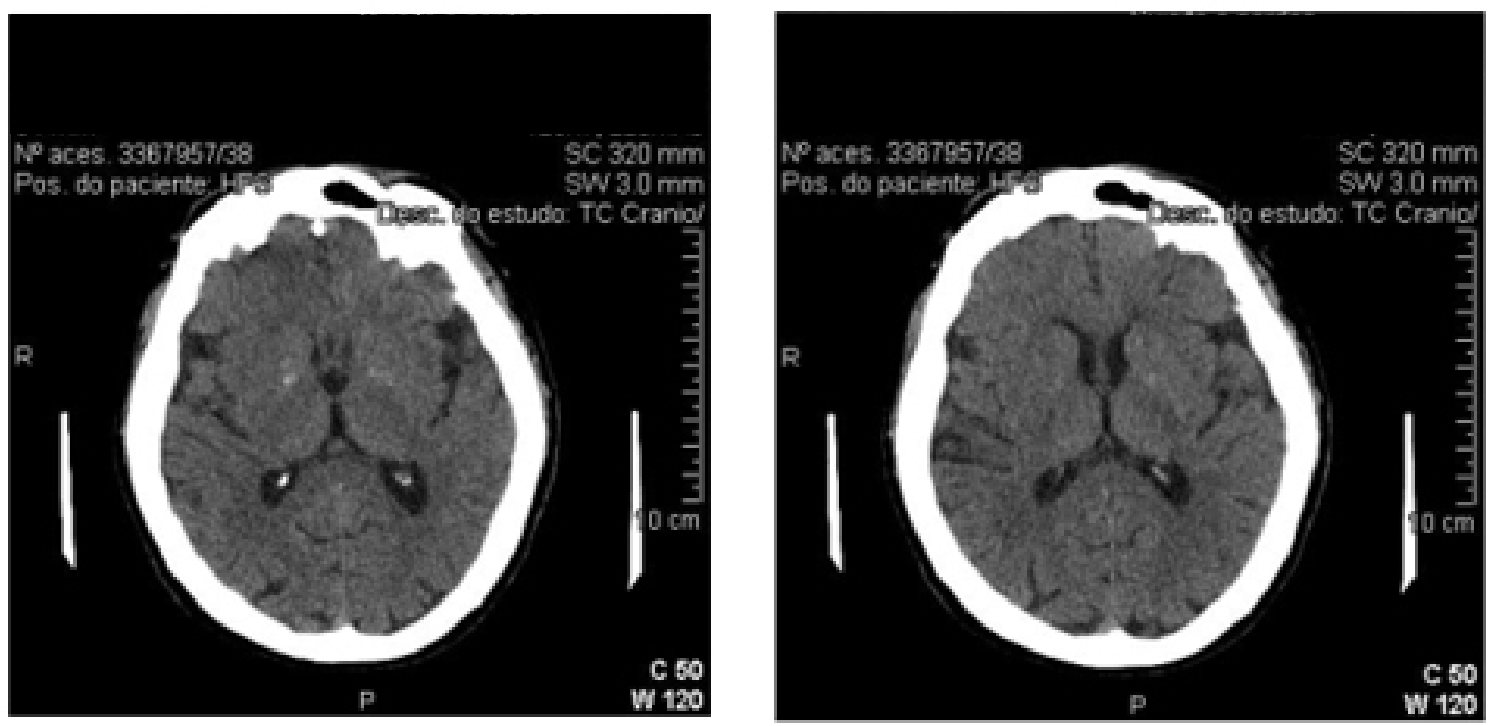

Figura 1. Tomografia Computadorizada de crânio sem contraste com 4 horas do início do ictus.
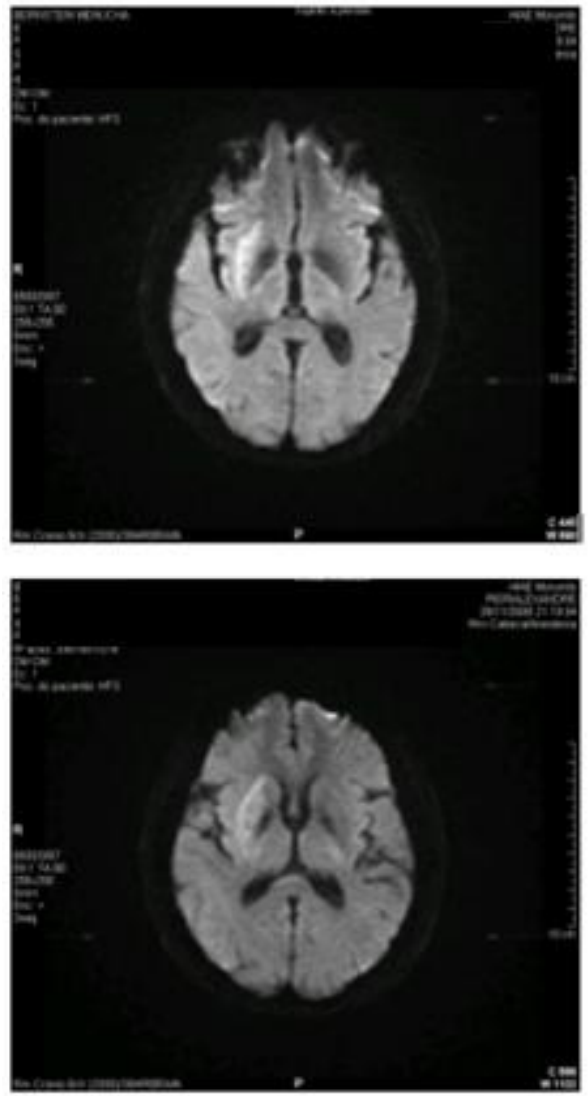

Figura 2. Ressonância Nuclear Magnética de encéfalo difusão com 4 horas e meia após o ictus.
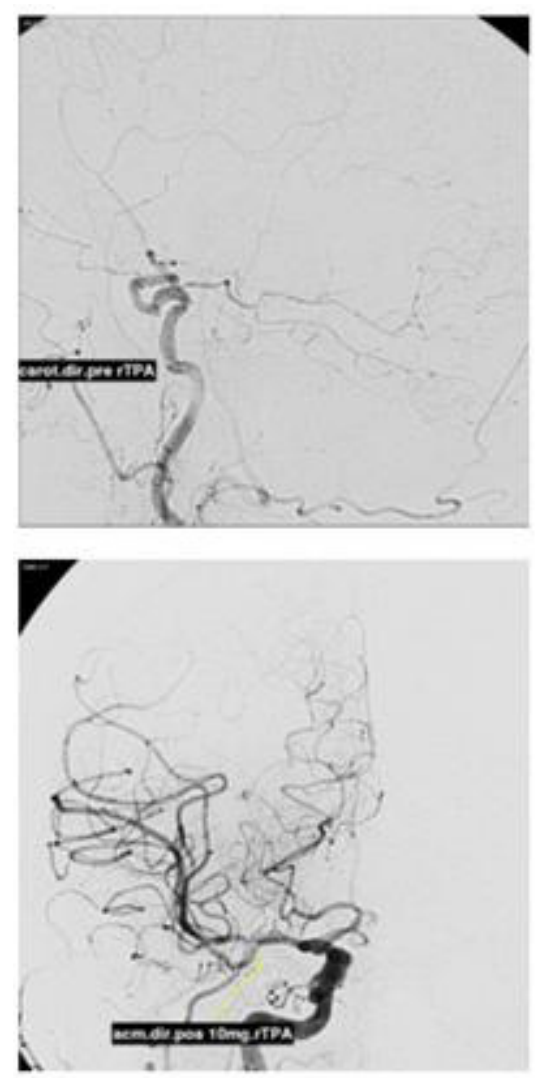

Figura 3. Angiografia cerebral pré e pós trombólise com cinco horas do ictus. Figura 4. Tomografia Computadorizada de crânio sem contraste após trombólise intra-arterial. 

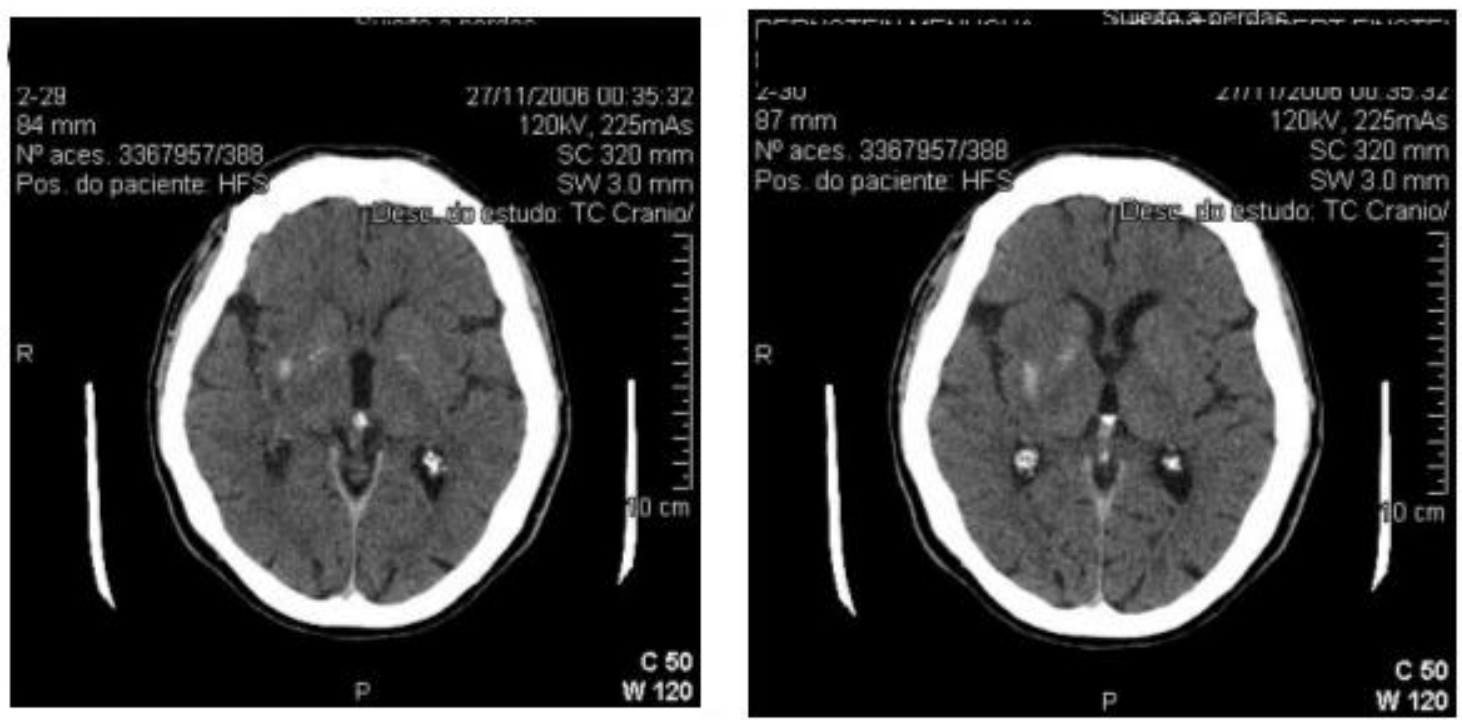

Figura 4. Tomografia Computadorizada de crânio sem contraste após trombólise intra-arterial.

Nas primeiras 24 horas em terapia intensiva fez ecocardiograma transesofágico sem evidências de trombos no átrio esquerdo, optando-se por cardioversão e manutenção de amiodarona e heparinização contínuas endovenosas com sucesso. Feito controle intensivo da PA, glicemia e temperatura. Foi realizada tomografia computadorizada de crânio sem contraste (Figura 5) que mostrou pequena lesão profunda de hemisfério direito

Nas primeiras 48 horas em terapia intensiva, a paciente desenvolveu pneumonia sendo medicada com clindamicina e ceftriaxone. Foi retirada a sedação e noradrenalina gradualmente e reavaliada. Ao exame apresentava-se com discreto desvio do olhar para direita, hemiparesia grau 2 à esquerda, hemianopsia homônima à esquerda, hemihipoestesia à esquerda e disartria.

Evoluiu com melhora clínica e neurológica importante recebendo alta da terapia intensiva após 8 dias e alta hospitalar com 16 dias de internação com escala de NIH de 4 e Rankim 19. Mantinha hemiparesia grau 4 à esquerda completa proporcionada, hemianopsia homônima à esquerda e disartria leve.

\section{DISCUSSÃO}

A monitorização da PA deve ser intensiva na fase aguda do AVCI para evitar complicações como hemorragias, principalmente nos casos elegíveis a trombólise sistêmica ou intra-arterial ${ }^{3}$.
A indicação de manutenção dos níveis de PA média na fase aguda do AVCl entre 90 e 110 $\mathrm{mmHg}$ melhora a morbidade e mortalidade desses pacientes. Os pacientes com PA sistólica menor que $90 \mathrm{mmHg}$ podem se beneficiar do uso de drogas vasoativas, particularmente em pacientes com lesão de córtex de insula à direita ${ }^{5,10}$. A dificuldade no controle de auto-regulação cerebral em pacientes com esse tipo de lesão predispõe à vasoparesia cerebral e sistêmica com dificuldade de manter a perfusão cerebral em áreas de penumbra.

Embora a droga vasoativa mais utilizada para manter os níveis pressóricos na fase aguda do AVCI seja a fenilefrina (pouco utilizada no nosso País), a opção pelo uso da noradrenalina, neste caso em particular, foi feita pelo maior acesso ao fármaco e pela maior experiência no manejo da droga6. Existem poucos relatos do uso de noradrenalina como droga para induz hipertensão ou para manter a PA na fase aguda do $\mathrm{AVCl}^{6,7}$.

\section{CONCLUSÃO}

Concluímos que embora poucos estudos tenham sido realizados, o uso da noradrenalina contribuiu positivamente na manutenção da $\mathrm{PA}$, neste caso, favorecendo a perfusão cerebral adequada pós trombólise intra-arterial. 

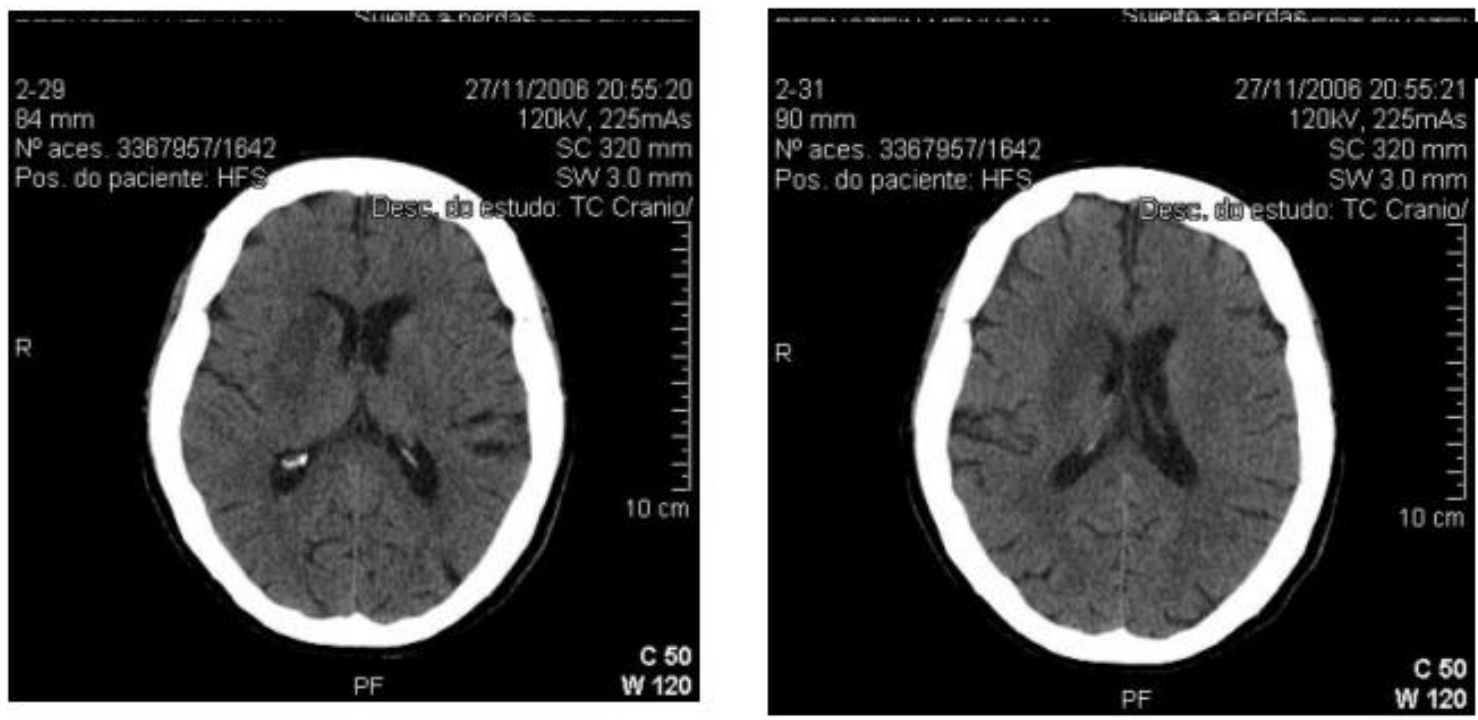

Figura 5. Tomografia Computadorizada de crânio sem contraste após 24 horas do ictus.

\section{REFERÊNCIAS BIBLIOGRÁFICAS}

1.Slama M, Modeliar SS. Hypertension in the intensive care unit. Curr Opin Cardiol 2006;21(4):279-87. 2.Semplicini A, Benetton V, Mascagna V, Macchini $L$, Realdi $A$, Sartori $M$, et al. Problems related to short-term antihypertensive therapy in acute ischemic stroke. Clin Exp Hypertens 2006;28(34):327-34.

3.Koenig MA, Geocadin RG, de Grouchy M, Glasgow J, Vimal S, Restrepo L, et al. Safety of induced hypertension therapy in patients with acute ischemic stroke. Neurocrit Care 2006;4(1):3-7.

4.Gagliardi RJ, Raffin CN, Fábio SRC, Bacellar A, Longo AL, Massaro AR, et al. Sociedade Brasileira de Doenças Cerebrovasculares. $1^{\circ}$ Consenso Brasileiro do Tratamento da Fase Aguda do Acidente Vascular Cerebral. Arq Neuropsiquiatr 2001;59:972-80. 5.Wityk RJ, Lewin JJ 3rd. Blood pressure management during acute ischemic stroke. Expert Opin Pharmacother 2006;7(3):247-58.
6.Rordorf G, Cramer SC, Efird JT, Schwamm LH, Buonanno F, Koroshetz WJ. Pharmacological elevation of blood pressure in acute stroke. Stroke 1997;28(11):2133-8.

7.Morfis L, Schwartz R, Lykos D, Zagami A, Pryor D, Howes LG. 24 hour ambulatory blood pressure profiles in the acute phase of stroke. Clin Exp Pharmacol Physiol 1995;22(10):775-7.

8.Spilker J, Kongable G, Barch C, Braimah J, Brattina P, Daley S, et al. Using the NIH Stroke Scale to assess stroke patients. The NINDS rt-PA Stroke Study Group. J Neurosci Nurs 1997;29(6):384-92.

9. Hamrin E, Wohlin A. Evaluation of the functional capacity of stroke patients through an activity index. Scand J Rehabil Med 1982;14(3):93-100.

10.Adams HP, Adams RJ, Brott T, del Zoppo GJ, FurIan A, Goldstein LB, et al. Guidelines for the early management of patients with ischemic stroke: a scientific statement from the Stroke Council of the American Stroke Association. Stroke 2003;34(4):1056-83. 